\title{
Dephosphorylation of NSSR1 regulates alternative splicing of the GluR-B minigene
}

\author{
W. Zhang ${ }^{1}$, L.L. Li ${ }^{1}$, X.H. Chen ${ }^{2}$ and Z.Y. Peng ${ }^{3}$ \\ ${ }^{1}$ School of Medicine, Zhejiang University City College, Hangzhou, China \\ ${ }^{2}$ State Key Laboratory of Medical Neurobiology, \\ Institute of Brain Science, Fudan University, Shanghai, China \\ ${ }^{3}$ Institute of Biomedical Sciences, Fudan University, Shanghai, China \\ Corresponding authors: Z.Y. Peng / W. Zhang \\ E-mail: zhypeng@fudan.edu.cn / zhangw@zucc.edu.cn
}

Genet. Mol. Res. 13 (1): 1753-1763 (2014)

Received November 30, 2012

Accepted August 10, 2013

Published March 17, 2014

DOI http://dx.doi.org/10.4238/2014.March.17.3

\begin{abstract}
Neural salient serine/arginine-rich protein 1 (NSSR1, alternatively SRp38) is an important splicing factor that can repress pre-mRNA alternative splicing in cells during heat shock and mitosis. We show here that NSSR1 protein is dephosphorylated when cells are heat shocked or incubated with kinase inhibitor K252a. Both heat shock and K252a treatment increase the truncated splicing isoform of the GluR-B minigene pre-mRNA. We also investigated the roles of the RRM motif and three RS domains of NSSR1 in in vivo pre-mRNA splicing. The results show that deletion of the RRM motif did not affect GluR-B minigene pre-mRNA splicing, but deletion of any one of the three RS domains increases the truncated splicing isoform of the GluR-B minigene. We further show that an SRSRSK sequence in the RS3 domain may play an important role in the function of NSSR1 in pre-mRNA splicing.
\end{abstract}

Key words: NSSR1; GluR-B minigene; Alternative splicing; Deletion; Dephosphorylation 


\section{INTRODUCTION}

Alternative splicing is one way of generating multiple products from a single gene at the post-transcriptional level. A recent survey of alternative splicing using exon junction microarrays suggests that $92-94 \%$ of human multiexon genes are alternatively spliced (Wang et al., 2008). Serine/arginine-rich (SR) proteins are a family of conserved, essential pre-mRNA splicing factors. SR proteins are required for basic, constitutive pre-mRNA splicing (Zahler et al., 1992), as well as for numerous alternative splicing events (Caceres et al., 1997). SR proteins have RNA binding domains (RRM domains) at the N-terminus, and several arginine/ serine-rich domains (RS domain) at the C-terminus. They regulate alternative splicing by interacting with U1-70k and U2AF35, which determine $5^{\prime}$ and $3^{\prime}$ splicing sites, respectively (Cao and Garcia-Blanco, 1998; Graveley, 2000). Phosphorylation of SR proteins plays important roles in alternative splicing, and also is necessary for entry of SR proteins into the nucleus, mRNA export, and other processing events (Ghosh and Adams, 2011). The RS domains are thought to serve as critical protein-protein adaptors, and to undergo rounds of phosphorylation and dephosphorylation during spliceosome maturation. Two protein kinase families, SR protein kinases and cdc2-like kinase play vital roles in phosphorylating SR proteins and controlling functions (Ngo et al., 2005; Ma et al., 2010). Previous studies have shown that specific phosphatases such as protein phosphatase 1 are able to dephosphorylate SR proteins and affect in vitro splicing (Cardinali et al., 1994; Cao et al., 1997). Phosphatase inhibitors have also been used to demonstrate that dephosphorylation is important for single splicing reactions (Mermoud et al., 1992). However, there are still insufficient data, and the mechanism underlying this complex reaction needs to be further elucidated.

Neural salient serine/arginine-rich protein 1 (NSSR1, also named SRp38) is an SR protein with a typical structure that includes one RRM motif and three RS domains (Komatsu et al., 1999). It was first identified in a yeast two-hybrid screen for proteins interacting with translocated in liposarcoma/fusion protein and named TASR (Yang et al., 1998, 2000a) and then discovered independently by Kotmasu (1999) and Shin and Manley (2002) as NSSR1 and SRp38, respectively. As an SR protein, NSSR1 has also been shown to regulate in vivo splicing of the GluR-B subunit of the AMPA receptor, tyrosine kinase receptor C, neural cell adhesion molecule L1, collagen, and cyclic AMP response element binding protein (CREB) (Komatsu et al., 1999; Clinton et al., 2002; Liu et al., 2004; Matsushita et al., 2007; Xiao et al., 2007). In in vitro experiments, the splicing of many pre-mRNAs, such as $\beta$-globin, HIVTAT, and IgM, has been found to be regulated by NSSR1 (Cowper et al., 2001). It can bind both RNA polymerase II and splicing factors, suggesting a link between transcription and splicing, and a potential role in aberrant splicing during carcinogenesis (Yang et al., 2000b; Shin and Manley, 2002). Its role of inhibiting neurogenesis during embryogenesis infers a potential role in nervous system development (Liu and Harland, 2005). However, some unusual properties of NSSR1 have been demonstrated that distinguish it from other SR proteins, e.g., during the M phase of mitosis, NSSR1 is dephosphorylated as well as activated as a splicing repressor (Shin and Manley, 2002). The dephosphorylated NSSR1 can also accumulate during heat shock when it inhibits splicing at an early step (Shin et al., 2004). Heat shockinduced NSSR1 dephosphorylation was found to display splicing thermotolerance mediated by the Hsp27 protein (Shi et al., 2011). Recently, we found that NSSR1 is expressed in mouse reproductive tissues such as the uterus and epididymis and, more interestingly, that the level 
of de-phosphorylated NSSR1 proteins in the mouse caput is upregulated during the first 3 weeks after birth, and was induced by androgen in the mouse epididymis (Peng et al., 2011; Xiao et al., 2011), indicating the potential roles that dephosphorylated NSSR1 protein might play during development of the epididymis and sperm maturation. It therefore appears that dephosphorylated NSSR1 proteins are involved in multiple aspects of physiological functions in different tissues and cells, including splicing, and that more functions need to be assigned to dephosphorylated NSSR1.

In this study, we inspected the role of dephosphorylated NSSR1 in regulating the GluR-B minigene during heat shock, and detected the role that the kinase inhibitor K252a plays in the phosphorylation status of NSSR 1 as well as its functions in splicing. Furthermore, we used deletion mutagenesis to study the roles of the RRM motif and RS domains of NSSR1 in regulating GluR-B minigene splicing.

\section{MATERIAL AND METHODS}

\section{Plasmid construction and transient transfection}

pcDNA3.1-NSSR1 and pDS-NSSR1 plasmids were constructed for overexpression and silencing of NSSR1, respectively, as previously described (Liu et al., 2003), pcDNA3.1 was used as the control. The GluR-B minigene was constructed as previously described (Chen et al., 2004). Transient transfection of cultured PFSK cells with pcDNA3.1-NSSR1 (or pDSNSSR1, or the control) and GluR-B minigene plasmids were conducted using Lipofectamine 2000 (Invitrogen), according to the manufacturer protocol. It was used $3.2 \mu \mathrm{g}$ of each plasmid for each $15-\mathrm{mm}$ culture dish.

\section{Cell culture, heat shock, and incubation with K252a}

PFSK cells were obtained from the American Type Culture Collection, and were primarily cultured as previously described, but with minor modification. Cells were maintained in RPMI-1640 medium (Invitrogen) supplemented with 10\% fetal bovine serum (Gibco). All cell cultures were incubated at $37^{\circ} \mathrm{C}$ with $5 \% \mathrm{CO}_{2}$. Spent medium was replaced every 2 to 3 days, and 1 day before use of the cells. Confluent cells were mixed $1: 3$ with $0.25 \%$ trypsin-EDTA to release the cells. Cells were subcultured in $15-\mathrm{mm}$ culture dishes for $24 \mathrm{~h}$ before assay.

Twenty-four hours after transfection, cells were heat shocked for $3 \mathrm{~h}$ at $41^{\circ} \mathrm{C}$ before harvesting. Kinase inhibitor K252a (Calbiochem, San Diego, CA, USA) was added at $100 \mathrm{nM}$ final concentration when renewing the growth medium $5 \mathrm{~h}$ after transfection. After incubation with K252a for $24 \mathrm{~h}$, cells were harvested for RT-PCR or Western blot analysis.

\section{RT-PCR analysis}

$\beta$-actin and GluR-B transcripts were analyzed by RT-PCR using the primers described previously (Chen et al., 2004). The transcripts were amplified for 32 cycles for GluR-B and 25 cycles for $\beta$-actin. PCR products were electrophoresed on $1 \%$ agarose gels with the same loading volume of each sample. After EtBr staining, the gels were photographed. DNA sequence analysis was performed to confirm the PCR products. 


\section{Western blot}

For Western blot analysis, the samples were boiled for $5 \mathrm{~min}$ before electrophoresis on a $12 \%$ polyacrylamide gel. The proteins were then transferred to a nitrocellulose membrane. After incubation for $1 \mathrm{~h}$ in 5\% instant non-fat milk powder in TBS to block non-specific binding, the membrane was incubated overnight at $4{ }^{\circ} \mathrm{C}$ with rabbit anti-NSSR1 antiserum (1:500 in blocking buffer), as described previously (Liu et al., 2003). The secondary antibody (APlabeled, 1:500 in blocking buffer) was applied for $2 \mathrm{~h}$. TBS containing $0.1 \%$ Tween was used to wash the membrane three times after incubation. Immunoreactive bands were visualized using BCIP/NBT (Watson) according to manufacturer instructions.

\section{Mutation construction}

pcDNA3.1-NSSR1 plasmid was used as the template to construct deletions. Mutations were designed and constructed as shown in Figure 1. The primers for each mutation are listed in Table S1.

\section{A}

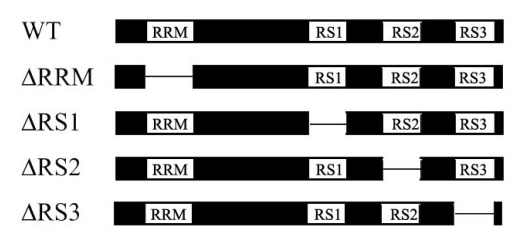

B

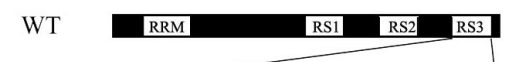

PRSKSQ SRSQ SRSRSK SRSRS

RS3 3 a PRSKSQ SRSQ SRSRSK

$\mathrm{RS} 3 \triangle \mathrm{b}$ PRSKSQ SRSQ

$\mathrm{RS} 3 \Delta \mathrm{c}$ PRSKSQ

$\mathrm{C}$
RS3 $\triangle$ al PRSKSQ SRSQ SRSRSK
$\triangle \mathrm{a} 2$ PRSKSQ SRSQ $*$ RSRSK
$\triangle \mathrm{a} 3$ PRSKSQ SRSQ $\mathrm{S} * \mathrm{SRSK}$
$\triangle \mathrm{a} 4$ PRSKSQ SRSQ SR $*$ RSK
$\triangle \mathrm{a} 5$ PRSKSQ SRSQ SRS $*$ SK
$\triangle \mathrm{a} 6$ PRSKSQ SRSQ SRSR $* \mathrm{~K}$

Figure 1. Diagram of the construction of deletion mutations. A. Diagram illustrating the deletions of RRM motif and three RS domains. The part of deletion is shown in a single line. B. Diagram illustrating the deletions of three part of RS3. C. Diagram illustrating the point mutation of RS3. 


\section{Statistical treatment}

Experiments were performed in triplicate and repeated at least three times independently. Data are reported as means $\pm \mathrm{SD}$, and the corresponding significance examined by the Student $t$-test.

\section{RESULTS}

\section{Effects of heat shock and K252a on NSSR1 dephosphorylation}

Previous studies have demonstrated that NSSR1 protein in HeLa cells is dephosphorylated in response to heat shock and acts as a splicing repressor (Shin et al., 2004), indicating that NSSR1 may play a crucial role in cell survival under stress conditions. To begin to determine whether dephosphorylated NSSR1 can inhibit the alternative splicing of the GluR-B minigene, we first examined the phosphorylation status of NSSR1 after heat shock. Western blot analysis of heatshocked PFSK cells and the untreated control (Figure 2) showed that the dephosphorylated isoform of both overexpressed and endogenous NSSR1 increased after heat shock as compared with the control. As kinase inhibitor represses the phosphorylation of protein (Tamura et al., 2006), we used K252a, a microbial alkaloid isolated from the culture broth of Nocardiopsis, to incubate with PFSK cells (MacInnis et al., 2003). We found that K252a also increases the dephosphorylated isoform of both overexpressed and endogenous NSSR1. These results show that NSSR1 protein expressed in PFSK cell can be dephosphorylated in response to heat shock and by incubation with K252a.

A

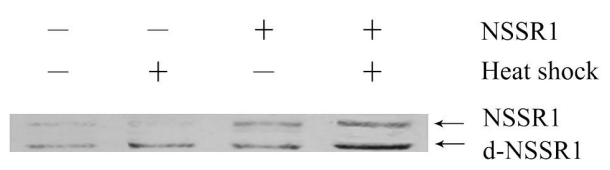

$\mathrm{C}$

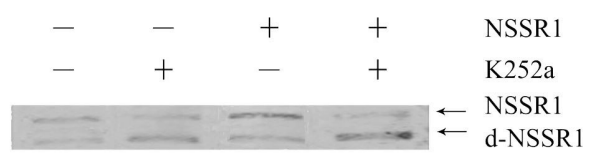

B

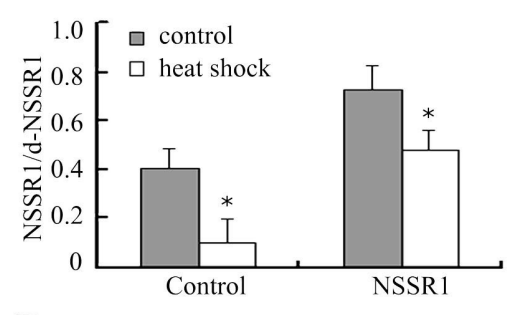

$\mathrm{D}$

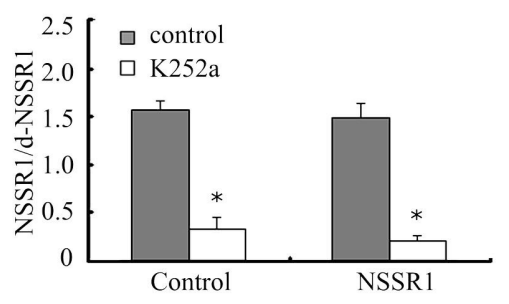

Figure 2. Effect of heat shock and K252a on regulating dephosphorylation status of NSSR1 in pcDNA3.1-NSSR1 transient transfected PFSK cells. A. Western blot analysis of phosphorylated (NSSR1) and dephosphorylated (d-NSSR1) NSSR1 after heat shock. pcDNA3.1 is used as the control. B. Statistical analysis of the ratio of d-NSSR1 to NSSR1 represented in A. Differences are considered to be statistically significant when $\mathrm{P}<0.01$ as indicated by stars $\left(^{*}\right) ; \mathrm{N}=4$. C. Western blot analysis of NSSR1 and d-NSSR1 after incubating the cells with K252a. pcDNA3.1-his-myc is used as the control. D. Statistical analysis of the ratio of d-NSSR1 to NSSR1 represented in A. Differences are considered to be statistically significant when $\mathrm{P}<0.01$ as indicated by stars $(*) ; \mathrm{N}=4$. 


\section{Effects of heat shock on GluR-B minigene splicing regulated by NSSR1}

As previous demonstrated, dephosphorylated NSSR1 can act as a general repressor of splicing in heat shocked cell extracts (Shin et al., 2004). In order to detect whether heat shock can inhibit GluR-B minigene pre-mRNA splicing in cultured cells, we first transiently co-transfected GluR-B minigene with the pcDNA3.1-NSSR1 plasmid into PFSK cells. According to the results with NIH3T3 cells (Komatsu et al., 1999), NSSR1 can also increase the flip exon inclusion of the GluR-B minigene in PFSK cells (Figures 3 and 4). After heat shock at $41^{\circ} \mathrm{C}$ for $3 \mathrm{~h}$, we found that the splicing pattern of the GluR-B minigene was dramatically changed in both NSSR1 overexpressing PFSK cells and control PFSK cells, with the flip exon inclusion decreased and the truncated isoform increased. Our results demonstrated that dephosphorylated NSSR1 caused by mild heat shock might only partially affect pre-mRNA splicing in in vivo analysis, compared with in vitro splicing analysis, which showed that dephosphorylated NSSR1 completely inhibits pre-mRNA splicing (Shin and Manley, 2002; Shin et al., 2004).

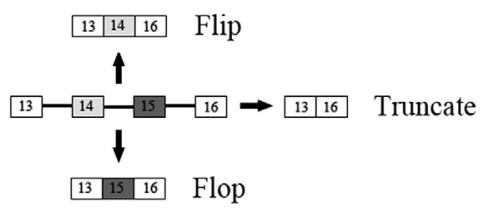

Figure 3. Diagram of the alternative splicing of GluR-B minigene. In cultured PFSK cells, the production of alternative splicing was identified by enzymatic digestion and proved to be flip and truncated except flop as previously described (Chen et al., 2004).

A

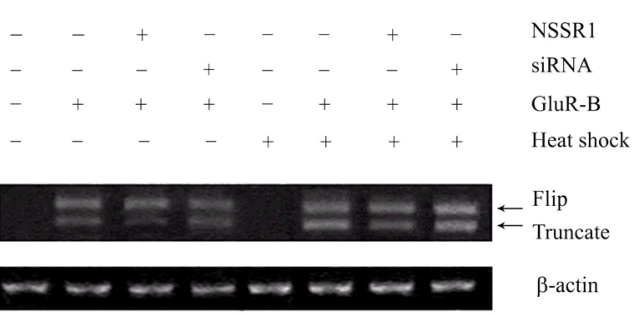

$\mathrm{B}$

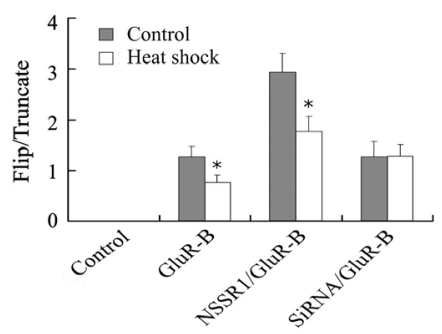

Figure 4. Effect of NSSR1 on alternative splicing of GluR-B minigene after heat shock. A. RT-PCR analysis shows the splicing of GluR-B minigene in PFSK cells with overexpressed (pcDNA3.1-NSSR1) or silenced (pDS-NSSR1) NSSR1 compared to control (pcDNA3.1). B. Statistical analysis of the ratio of GluR-B (flip plus truncated) to $\beta$-actin represented by A. Differences are considered to be statistically significant when $\mathrm{P}<0.01$ as indicated by stars $(*) ; \mathrm{N}=4$. C. Statistical analysis of the ratio of flip to truncated represented by $\mathbf{A}$. Differences are considered to be statistically significant when $\mathrm{P}<0.01$ as indicated by stars $(*) ; \mathrm{N}=4$. 


\section{Effects of K252a on GluR-B minigene splicing regulated by NSSR1}

We also examined the effect of K252a on the regulation of NSSR1 in GluR-B minigene splicing. After incubating the cells with K252a for $24 \mathrm{~h}$, our results show that the splicing pattern of GluR-B minigene was changed in both NSSR1 overexpressing PFSK cells and the control PFSK cells, with the flip exon inclusion decreased and the truncated isoform increased. However, similarly to $\beta$-actin, K252a did not inhibit GluR-B minigene splicing, as shown in Figure 5.

A

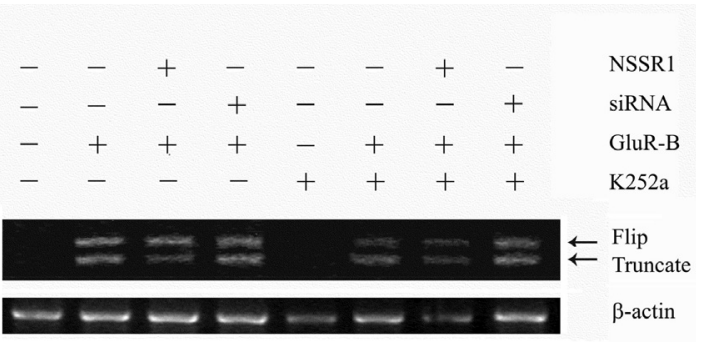

B

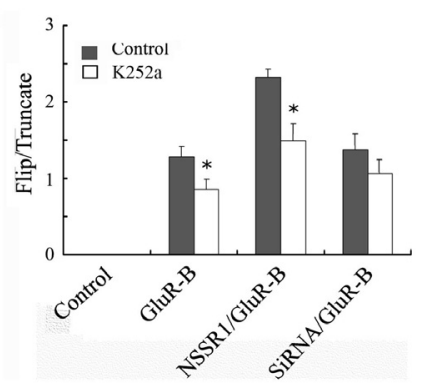

Figure 5. Effect of NSSR1 on alternative splicing of GluR-B minigene after incubated with K252a. A. RT-PCR analysis shows the splicing of GluR-B minigene in PFSK cells transfected with pcDNA3.1-NSSR1 or pDS-NSSR1 compared to control (pcDNA3.1). B. Statistical analysis of the ratio of GluR-B (flip plus truncated) to $\beta$-actin represented by $\mathbf{A}$. Differences are considered to be statistically significant when $\mathrm{P}<0.01$ as indicated by stars $(*)$; $\mathrm{N}=4$. C. Statistical analysis of the ratio of flip to truncated represented by A. Differences are considered to be statistically significant when $\mathrm{P}<0.01$ as indicated by stars $(*) ; \mathrm{N}=4$.

\section{RS domains of NSSR1 play roles on GluR-B minigene splicing}

When we co-transfected cells with the GluR-B minigene and pcDNA3.1-NSSR1 from which the RRM motif or the three RS domains had been deleted, we found that it did not change the function of NSSR1 in regulating alternative splicing, whereas deletion of each of the three RS domains of NSSR1 significantly increased the truncated isoform of the GluR-B minigene, as shown in Figures 1 and 6.

Since the main difference between NSSR1 and its homogenous isoform, NSSR2, is that NSSR2 lacks the whole RS3 and part of the RS2 domain, we further constructed partial deletions of RS3 and investigated their effects on the regulation function of NSSR1 in GluR-B minigene splicing. Our result show that deletion of fragment A (lacking SRSRS) from RS3 
did not influence the splicing pattern of the GluR-B minigene, but deletion of fragment B (lacking SRSRSKSRSRS) changed the splicing pattern significantly. The effect of fragment $C$ deletion, however, (SRSQSRSRSKSRSRS) was the same as that of the fragment B deletion. The results imply that the sequence of SRSRSK may be important to the splicing function of NSSR1. However, when we examined the effect of individual point mutations in SRSRSK individually, we could not detect any effect when compared with the control.

A

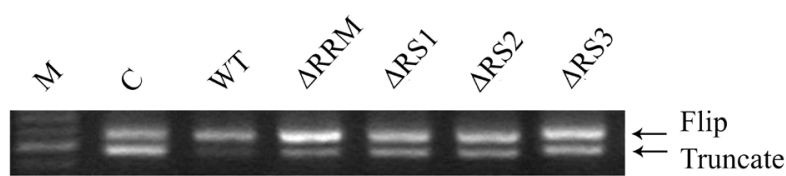

$\mathrm{B}$

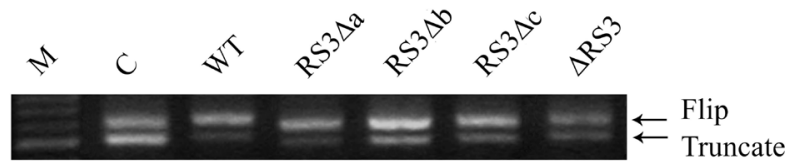

$\mathrm{C}$

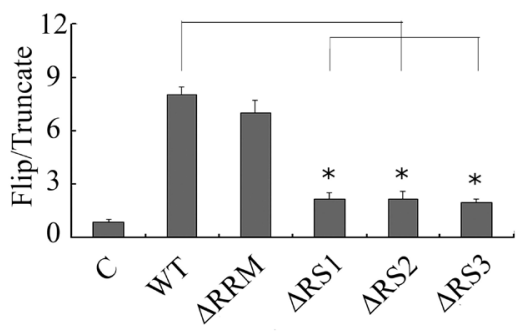

$\mathrm{D}$

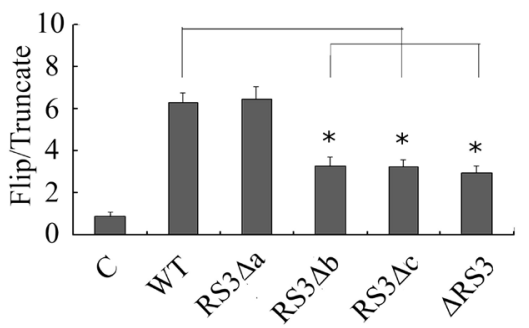

Figure 6. Effect of the deletion mutations of NSSR1 on alternative splicing of GluR-B minigene. A. RT-PCR analysis shows the splicing of GluR-B minigene in PFSK cells transfected with wild type (WT) or RRM and three RS mutations of NSSR1 (pcDNA3.1-NSSR1) compared to control (pcDNA3.1). B. RT-PCR analysis shows the splicing of GluR-B minigene in PFSK cells transfected with WT or three RS3 mutations of NSSR1 (pcDNA3.1NSSR1) compared to control (pcDNA3.1). C. Statistical analysis of the ratio of flip to truncated represented by A. Differences are considered to be statistically significant when $\mathrm{P}<0.01$ as indicated by stars $(*) ; \mathrm{N}=4$. D. Statistical analysis of the ratio of flip to truncated represented by $\mathbf{A}$. Differences are considered to be statistically significant when $\mathrm{P}<0.01$ as indicated by stars $(*) ; \mathrm{N}=4$.

\section{DISCUSSION}

It is well known that heat shock causes a global shut down of pre-mRNA splicing (Yost and Lindquist, 1986; Wright-Sandor et al., 1989). Furthermore, it is well established that mild heat shock prevents many of the deleterious effects (including inhibition of splicing) of severe heat shock via the induction and expression of heat shock proteins (Yost and Lindquist, 1986, 1991), but the molecular mechanisms have remained largely obscure. NSSR1 has been found as 
a splicing repressor upon dephosphorylation triggered by severe heat shock (Shin et al., 2004). A recent study shows that mild heat shock protects NSSR1 (SRSF10) from dephosphorylation during a second and more severe heat shock, and this splicing thermotolerance is mediated at least in part by Hsp27 (Shi et al., 2011). However, during mild heat shock, the roles NSSR1 plays in functions such as splicing are still largely obscure.

In our experiments, we found that the phosphorylation status of both endogenous and overexpressed NSSR1 changed, with dephosphorylated NSSR1 increasing significantly in PFSK cells exposed to $41^{\circ} \mathrm{C}$ for $3 \mathrm{~h}$. Our studies further demonstrated that, unlike severe heat shock, NSSR1 dephosphorylated by mild heat shock does not act as a general repressor of pre-mRNA splicing. Instead, it can regulate the splicing of the GluR-B minigene and increase the truncated isoform. Thus, these results support the possibility that, as an SR protein with typical structure, dephosphorylated NSSR1 may play different roles during mild and severe heat shock via different mechanisms. In our recent study, by Western blot analysis of NSSR1 expression in mouse cryptorchidism, we also demonstrated that there is a significant increase in dephosphorylated NSSR1 compared with normal testis, and this is accompanied by the morphological changes in cryptorchidism. In addition, the splicing of CREB in cryptorchidism is regulated by dephosphorylated NSSR1 (Xiao et al., 2007). Marin-Vinader et al. (2006) found that a small heat shock protein, Hsp27, enhances splicing recovery as well as rephosphorylation of NSSR1 after heat shock. However, the molecular mechanism of NSSR1 activity in mild heat shock conditions still needs to be demonstrated.

The phosphorylation status of an SR protein is controlled by phosphatases and kinases, as previously demonstrated (Jurica and Moore, 2003). Shin and Manley (2002) and Shin et al. (2004) found that phosphatase can dephosphorylate NSSR1. Other studies have shown that K252a, an inhibitor of many kinases including protein kinase C, phosphorylase kinase, and tyrosine kinase, can block NGF-induced neurite outgrowth and the changes in protein phosphorylation elicited by NGF (MacInnis et al., 2003; Tamura et al., 2006). In this study, we incubated cultured PFSK cells with K252a kinase inhibitor and found that it causes NSSR1 dephosphorylation. Furthermore, our results indicate that addition of K252a can also influence splicing of the GluR-B minigene in a similar way to heat shock. Therefore, heat shock and kinase inhibitor K252a may dephosphorylate NSSR1 through the same mechanism.

SR proteins are characterized by the presence of an RRM and a $\mathrm{COOH}$-terminal domain rich in arginine and serine residues (RS domain) (Zahler et al., 1992). The RRM determines RNA-binding specificity, while the RS domain functions as a protein-protein interaction module by recruiting components of the core splicing apparatus, such as U1-70k, to promote splice site pairing (Graveley, 2000). Arginine and serine residues in the RS domain are the main phosphorylation sites, and phosphorylation of the RS domain plays an important role in regulating pre-mRNA splicing and localization of SR proteins (Dagher and $\mathrm{Fu}, 2001$ ). It is reported that the RRM of NSSR1 can recognize mRNAs by inserting the stem loops of the MS2 RNA into the middle of the N exon of the clathrin light chain B minigene (Fushimi et al., 2005). However, Shin et al. (2005) found that a chimeric protein of the RRM of NSSR1 and the RS domains of SC35 cannot activate the splicing of $\beta$-globin pre-mRNA, whereas a chimeric protein of the RRM from SC35 and the RS domains of NSSR1 can activate the splicing. Consistent with this, we found that deletion of RRM from NSSR1 did not change the splicing of the GluR-B minigene. Amino acids 143-234 of NSSR1 have been shown by deletion analyses to contribute to the exon inclusion of the clathrin light chain B minigene, whereas the 
RS3 of NSSR1 was not necessary (Fushimi et al., 2005). However, our data revealed that the RS3 domain does plays a role in regulating splicing of the GluR-B minigene. As the main difference between NSSR1 and NSSR2 is that NSSR2 lacks RS3 and part of RS2, our results are consistent with previous results that NSSR2 can increase the truncated isoform of the GluR-B minigene (Komatsu et al., 1999). Combining this with results from earlier studies, we believe that the RS3 might have different roles in regulating the splicing of different minigenes. The "strength" of RS domains as activators reflects the number of RS dipeptides (Graveley, 2000). However, our further studies indicate that an SRSRSK sequence in the RS3 domain might be important in pre-mRNA splicing. More research is needed to verify the role this SRSRSK sequence plays in pre-mRNA splicing.

In conclusion, our study demonstrates that, although NSSR1 behaves unusually by inhibiting alternative splicing during severe heat shock or mitosis, as an SR protein with typical structure, dephosphorylated NSSR1 can regulate alternative splicing of the GluR-B minigene in PFSK cells during mild heat shock or treatment with kinase inhibitor. Using deletion mutagenesis, we found that the RRM did not influence alternative splicing directly, but the RS domains may participate in the process of alternative splicing, probably via protein-protein interactions between components of the spliceosome such as U1-70k.

\section{Supplementary material}

\section{REFERENCES}

Caceres JF, Misteli T, Screaton GR, Spector DL, et al. (1997). Role of the modular domains of SR proteins in subnuclear localization and alternative splicing specificity. J. Cell Biol. 138: 225-238.

Cao W and Garcia-Blanco MA (1998). A serine/arginine-rich domain in the human U1 70k protein is necessary and sufficient for ASF/SF2 binding. J. Biol. Chem. 273: 20629-20635.

Cao W, Jamison SF and Garcia-Blanco MA (1997). Both phosphorylation and dephosphorylation of ASF/SF2 are required for pre-mRNA splicing in vitro. RNA 3: 1456-1467.

Cardinali B, Cohen PT and Lamond AI (1994). Protein phosphatase 1 can modulate alternative 5' splice site selection in a HeLa splicing extract. FEBS Lett. 352: 276-280.

Chen X, Huang J, Li J, Han Y, et al. (2004). Tra2betal regulates P19 neuronal differentiation and the splicing of FGF-2R and GluR-B minigenes. Cell Biol. Int. 28: 791-799.

Clinton JM, Chansky HA, Odell DD, Zielinska-Kwiatkowska A, et al. (2002). Characterization and expression of the human gene encoding two translocation liposarcoma protein-associated serine-arginine (TASR) proteins. Gene 284: 141-147.

Cowper AE, Caceres JF, Mayeda A and Screaton GR (2001). Serine-arginine (SR) protein-like factors that antagonize authentic SR proteins and regulate alternative splicing. J. Biol. Chem. 276: 48908-48914.

Dagher SF and Fu XD (2001). Evidence for a role of Skylp-mediated phosphorylation in 3' splice site recognition involving both Prp8 and Prp17/Slu4. RNA 7: 1284-1297.

Fushimi K, Osumi N and Tsukahara T (2005). NSSRs/TASRs/SRp38s function as splicing modulators via binding to premRNAs. Genes Cells 10: 531-541.

Ghosh G and Adams JA (2011). Phosphorylation mechanism and structure of serine-arginine protein kinases. FEBS J. 278: 587-597.

Graveley BR (2000). Sorting out the complexity of SR protein functions. RNA 6: 1197-1211.

Jurica MS and Moore MJ (2003). Pre-mRNA splicing: awash in a sea of proteins. Mol. Cell 12: 5-14.

Komatsu M, Kominami E, Arahata K and Tsukahara T (1999). Cloning and characterization of two neural-salient serine/ arginine-rich (NSSR) proteins involved in the regulation of alternative splicing in neurones. Genes Cells 4: 593-606.

Liu KJ and Harland RM (2005). Inhibition of neurogenesis by SRp38, a neuroD-regulated RNA-binding protein. Development 132: 1511-1523.

Liu L, Lin JJ, Chen X, Liu X, et al. (2003). Neural expression and regulation of NSSR1 proteins. Neuroreport 14: 18471850. 
Liu L, Chen XH, Huang J, Lin JJ, et al. (2004). NSSR1 promotes neuronal differentiation of mouse embryonic carcinoma P19 cells. Neuroreport 15: 823-828.

Ma CT, Ghosh G, Fu XD and Adams JA (2010). Mechanism of dephosphorylation of the SR protein ASF/SF2 by protein phosphatase 1. J. Mol. Biol. 403: 386-404.

MacInnis BL, Senger DL and Campenot RB (2003). Spatial requirements for TrkA kinase activity in the support of neuronal survival and axon growth in rat sympathetic neurons. Neuropharmacology 45: 995-1010.

Marin-Vinader L, Shin C, Onnekink C, Manley JL, et al. (2006). Hsp27 enhances recovery of splicing as well as rephosphorylation of SRp38 after heat shock. Mol. Biol. Cell 17: 886-894.

Matsushita H, Blackburn ML, Klineberg E, Zielinska-Kwiatkowska A, et al. (2007). TASR-1 regulates alternative splicing of collagen genes in chondrogenic cells. Biochem. Biophys. Res. Commun. 356: 411-417.

Mermoud JE, Cohen P and Lamond AI (1992). Ser/Thr-specific protein phosphatases are required for both catalytic steps of pre-mRNA splicing. Nucleic Acids Res. 20: 5263-5269.

Ngo JC, Chakrabarti S, Ding JH, Velazquez-Dones A, et al. (2005). Interplay between SRPK and Clk/Sty kinases in phosphorylation of the splicing factor ASF/SF2 is regulated by a docking motif in ASF/SF2. Mol. Cell 20: 77-89.

Peng ZY, Xiao PJ, Qi Y, Zhang W, et al. (2011). NSSR1 is regulated by testosterone in the mouse uterus and extensively expressed in endometrial carcinoma. Tumour Biol. 32: 359-366.

Shi Y, Nishida K, Campigli Di GD and Manley JL (2011). Heat shock-induced SRSF10 dephosphorylation displays thermotolerance mediated by Hsp27. Mol. Cell Biol. 31: 458-465.

Shin C and Manley JL (2002). The SR protein SRp38 represses splicing in M phase cells. Cell 111: 407-417.

Shin C, Feng Y and Manley JL (2004). Dephosphorylated SRp38 acts as a splicing repressor in response to heat shock. Nature 427: 553-558.

Shin C, Kleiman FE and Manley JL (2005). Multiple properties of the splicing repressor SRp38 distinguish it from typical SR proteins. Mol. Cell Biol. 25: 8334-8343.

Tamura M, Koyama R, Ikegaya Y, Matsuki N, et al. (2006). K252a, an inhibitor of Trk, disturbs pathfinding of hippocampal mossy fibers. Neuroreport 17: 481-486.

Wang ET, Sandberg R, Luo S, Khrebtukova I, et al. (2008). Alternative isoform regulation in human tissue transcriptomes. Nature 456: 470-476.

Wright-Sandor LG, Reichlin M and Tobin SL (1989). Alteration by heat shock and immunological characterization of Drosophila small nuclear ribonucleoproteins. J. Cell Biol. 108: 2007-2016.

Xiao PJ, Hu L, Li J, Lin W, et al. (2007). NSSR1 is regulated in testes development and cryptorchidism and promotes the exon 5-included splicing of CREB transcripts. Mol. Reprod. Dev. 74: 1363-1372.

Xiao PJ, Peng ZY, Huang L, Li Y, et al. (2011). Dephosphorylated NSSR1 is induced by androgen in mouse epididymis and phosphorylated NSSR1 is increased during sperm maturation. PLoS One 6: e25667.

Yang L, Embree LJ, Tsai S and Hickstein DD (1998). Oncoprotein TLS interacts with serine-arginine proteins involved in RNA splicing. J. Biol. Chem. 273: 27761-27764.

Yang L, Chansky HA and Hickstein DD (2000a). EWS.Fli-1 fusion protein interacts with hyperphosphorylated RNA polymerase II and interferes with serine-arginine protein-mediated RNA splicing. J. Biol. Chem. 275: 37612-37618.

Yang L, Embree LJ and Hickstein DD (2000b). TLS-ERG leukemia fusion protein inhibits RNA splicing mediated by serine-arginine proteins. Mol. Cell Biol. 20: 3345-3354.

Yost HJ and Lindquist S (1986). RNA splicing is interrupted by heat shock and is rescued by heat shock protein synthesis. Cell 45: 185-193.

Yost HJ and Lindquist S (1991). Heat shock proteins affect RNA processing during the heat shock response of Saccharomyces cerevisiae. Mol. Cell Biol. 11: 1062-1068.

Zahler AM, Lane WS, Stolk JA and Roth MB (1992). SR proteins: a conserved family of pre-mRNA splicing factors. Genes Dev. 6: 837-847. 